\title{
A Numerical Investigation of the Effect of Cubic Crystals Orientation on the Indentation Modulus
}

\author{
A. MATERnA ${ }^{a, *}$, P. HAUŠILD $^{a}$ AND J. NOHAVA ${ }^{b}$ \\ ${ }^{a}$ Czech Technical University in Prague, Faculty of Nuclear Sciences and Physical Engineering, \\ Department of Materials, Trojanova 13, 12000 Praha 2, Czech Republic \\ ${ }^{b}$ Anton Paar TriTec SA, Rue de la Gare 4, CH-2034 Peseux, Switzerland
}

\begin{abstract}
The elastic finite element computations of the indentation process with the Berkovich indenter are performed to examine the effect of cubic crystal and indenter orientation on indentation moduli of anisotropic material. Three metals with a cubic crystal lattice and various degree of elastic anisotropy from 1 to 9 are studied: tungsten, AISI 304 steel and $\beta$-brass. Differences in anisotropy ratios expressed by the Young moduli on the one side and by the indentation moduli on the other side are quantified.
\end{abstract}

DOI: 10.12693/APhysPolA.128.693

PACS: $62.20 . \mathrm{de}$

\section{Introduction}

Indentation is often the only way to determine the mechanical properties of a small volume of material, e.g. the Young modulus of thin films. Although many untextured polycrystalline materials are isotropic at macroscale, at micro- or nanoscale they exhibit anisotropic mechanical behaviour. In a single crystal, the elastic properties often differ with orientation.

In our previous study [1] we performed the nanoindentation of AISI 304 stainless steel (SS) at the scale of a single grain together with characterization of its orientation by the electron backscattered diffraction. We found the strong correlation between the indentation modulus and orientation of the grain, but the anisotropy ratio was much lower than that expressed by the $E_{111} / E_{100}$ ratio. Subsequent finite element analysis of the indentation process $[1,2]$ verified the conclusion made from experimental observations.

In this paper, extended numerical studies of indentation processes of three metals with cubic crystal lattice and various degree of elastic anisotropy are performed.

\section{Cubic crystal anisotropy}

The resistance of a crystal to elastic deformation is strongly dependent on its orientation and can be expressed by the generalized Hook law in the matrix form

$$
\sigma=C \varepsilon,
$$

where $\sigma, \varepsilon$, and $C$ are stress, strain and stiffness matrix, respectively.

For cubic crystal symmetry, only 3 independent coefficients $C_{11}, C_{12}$ and $C_{44}$ are needed and Eq. (1) is reduced to

${ }^{*}$ corresponding author; e-mail: ales.materna@fjfi.cvut.cz

$$
\left[\begin{array}{c}
\sigma_{11} \\
\sigma_{22} \\
\sigma_{33} \\
\tau_{23} \\
\tau_{13} \\
\tau_{12}
\end{array}\right]=\left[\begin{array}{cccccc}
C_{11} & C_{12} & C_{12} & 0 & 0 & 0 \\
C_{12} & C_{11} & C_{12} & 0 & 0 & 0 \\
C_{12} & C_{12} & C_{11} & 0 & 0 & 0 \\
0 & 0 & 0 & C_{44} & 0 & 0 \\
0 & 0 & 0 & 0 & C_{44} & 0 \\
0 & 0 & 0 & 0 & 0 & C_{44}
\end{array}\right]\left[\begin{array}{c}
\varepsilon_{11} \\
\varepsilon_{22} \\
\varepsilon_{33} \\
\gamma_{23} \\
\gamma_{13} \\
\gamma_{12}
\end{array}\right] .
$$

The Young modulus in an arbitrary direction $h k l$ can be calculated using the following equation:

$$
\begin{aligned}
& \frac{1}{E_{h k l}}=\frac{C_{11}+C_{12}}{\left(C_{11}-C_{12}\right)\left(C_{11}+2 C_{12}\right)} \\
& -\left(\frac{2}{C_{11}-C_{12}}-\frac{1}{C_{44}}\right)\left(l^{2} m^{2}+m^{2} n^{2}+l^{2} n^{2}\right),
\end{aligned}
$$

where $l, m$, and $n$ are the direction cosines of the angle between the vector corresponding to the direction, and the $x, y$, and $z$ axes, respectively.

The anisotropy in elastic response can be characterized by the ratio of the Young moduli in the hard and soft direction $E_{111} / E_{100}$ or by the Zener anisotropy ratio $2 C_{44} /\left(C_{11}-C_{12}\right)$.

\section{Measurement of elastic modulus by instrumented nanoindentation}

The method for evaluation of elastic modulus of isotropic materials from indentation load-displacement $(P-h)$ data was introduced by Oliver and Pharr $[3,4]$. It assumes the measurement of the maximum load in the cycle $P_{\max }$, the maximum displacement $h_{\max }$ and the elastic unloading stiffness $S=d P / d h$ defined as the slope of the upper part of the unloading curve. Then contact area $A$ is evaluated (for details see original paper [4]). The effective elastic modulus which takes into account elastic deformation of the indenter is given by the relation

$$
E_{\text {eff }}=\frac{\sqrt{\pi}}{2 \beta} \frac{S}{\sqrt{A}},
$$

where correction factor $\beta$ is slightly greater than unity 
and accounts for e.g. non-axisymmetric shape of the indenter.

For anisotropic materials, the indentation modulus generally depends on the shape of the indenter, although it is independent of indenter geometry under certain conditions of crystal symmetry. For general anisotropic material authors in [5] defined indentation modulus $M$ through the equation

$$
M=\frac{\sqrt{\pi}}{2} \frac{S}{\sqrt{A}} .
$$

For isotropic materials and axisymmetric geometries $M$ reduces to the plane-strain elastic modulus $M=\frac{E}{1-\nu^{2}}$, independent of indenter shape.

\section{Numerical study}

The goal of the presented study was to predict the variation of the indentation moduli on the crystallographic orientations of various anisotropic grains. All numerical analyses were based on finite element method (FEM).

\subsection{Materials}

For the numerical study, three cubic crystal materials with various degree of anisotropy were chosen: tungsten, commercial AISI 304 grade stainless steel and $\beta$-brass. Their elastic properties taken from $[6,7]$ are summed up in the Table.

TABLE

Elastic stiffness constants and corresponding anisotropic ratios for three studied materials.

\begin{tabular}{c|c|c|c|c|c}
\hline \hline $\begin{array}{c}\text { Material } \\
\text { (source) }\end{array}$ & $\begin{array}{c}C_{11} \\
{[\mathrm{GPa}]}\end{array}$ & $\begin{array}{c}C_{12} \\
{[\mathrm{GPa}]}\end{array}$ & $\begin{array}{c}C_{44} \\
{[\mathrm{GPa}]}\end{array}$ & $\frac{E_{111}}{E_{100}}$ & $\frac{2 C_{44}}{C_{11}-C_{12}}$ \\
\hline $\mathrm{W}[6]$ & 522.4 & 204.4 & 160.8 & 1.01 & 1.01 \\
SS [7] & 209 & 133 & 121 & 2.74 & 3.18 \\
$\beta$-brass [6] & 126.5 & 107.7 & 80.3 & 7.11 & 8.54
\end{tabular}

\subsection{FEM model}

FEM nonlinear code MSC.Marc was used for numerical evaluation of the indentation modulus.

As the recovery of the indentation during unloading can be calculated from the theory of elasticity (considering the contact of an indenter with an elastic half space), only time-saving elastic analyses under small-strain condition were performed. However, it should be noted that so computed load-displacement curves are not directly comparable with actual experimental data.

The contact between the rigid Berkovich indenter and deformable material is considered frictionless due to very low tangential angle between sample and indenter tip.

The finite element mesh of indented material consist of 74850 eight-node linear brick elements and 35 six-node linear wedge elements placed directly under the indenter tip. The smallest elements with a size $c a .0 .1 \mu \mathrm{m}$ in the area of the supposed contact and its close surroundings
(Fig. 1) were adaptively subdivided in 3 steps to final size ca. $0.01 \mu \mathrm{m}$ (Fig. 2). The built mesh respects the geometry of the indenter - there are no nodes directly under the indenter edges. It minimizes problems with proper contact detection. The entire model was $c a .400 \mu \mathrm{m}$ in diameter and $200 \mu \mathrm{m}$ in height.

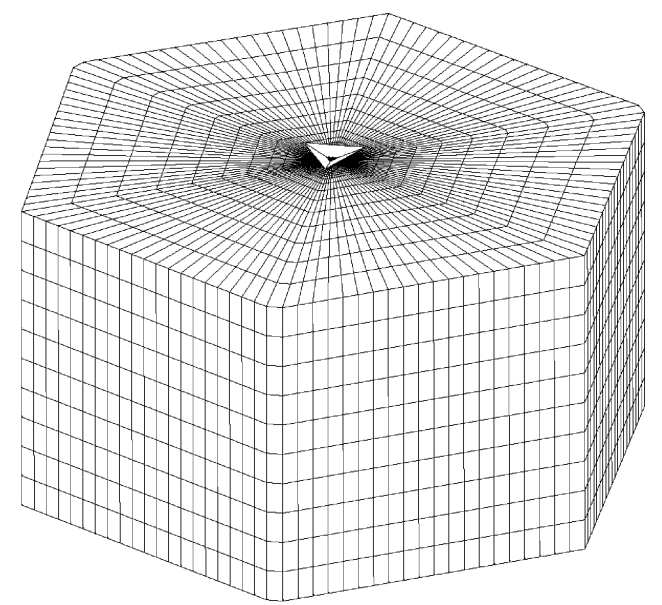

Fig. 1. Finite element model of the indented material and the rigid indenter.

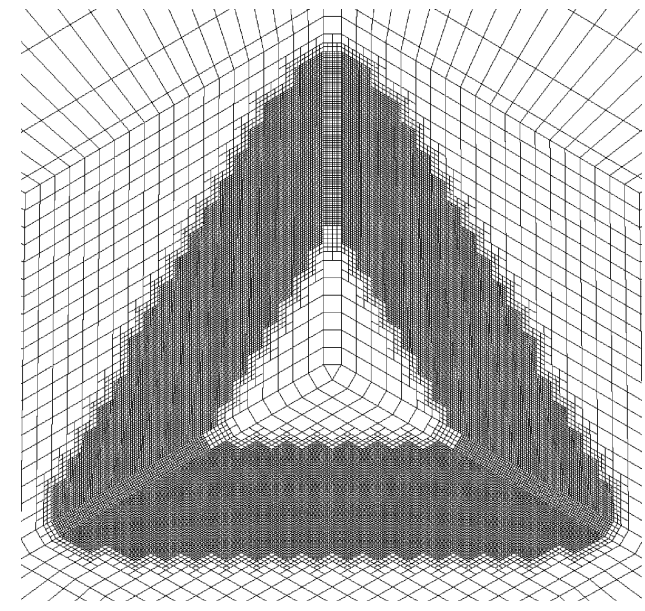

Fig. 2. Finite element mesh subdivision under the indenter.

The specimen was fixed on its bottom only, although the relatively large size of the specimen minimizes the effect of boundary conditions on its exterior on the achieved results. Indentation was driven by the displacement of the indenter with the maximum penetration depth $h_{\max }=0.5 \mu \mathrm{m}$.

\subsection{Indentation modulus determination}

The indentation modulus determination was based on the Oliver-Pharr method modified according to Eq. (5).

The contact area $A$ was evaluated directly from model nodes in contact with the surfaces of the indenter (Fig. 3). 
The exact boundary for the area evaluation was determined using the Matlab function for $\alpha$-shape detection based on the Delaunay triangulation.

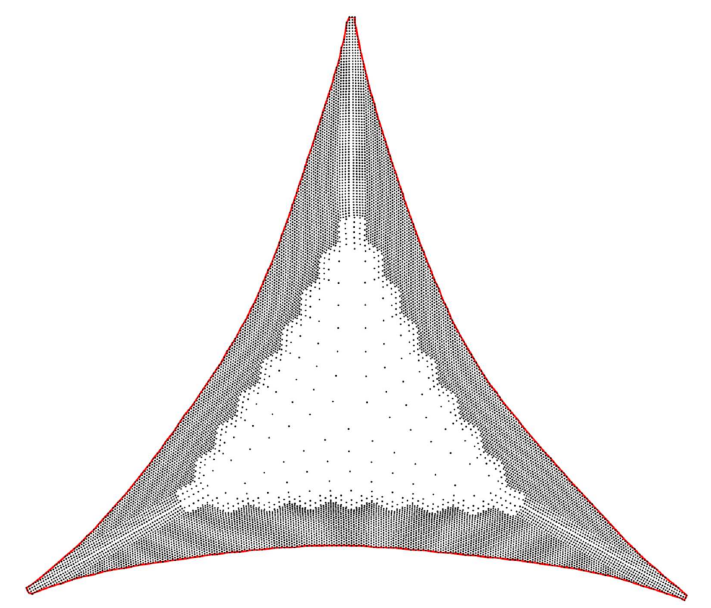

Fig. 3. Typical result showing nodes in contact with the indenter. Evaluated boundary line forms contact area.

\section{Results and discussion}

From ca. 400 numerical simulations comprising 3 various materials, and, for each 24 representatively chosen material crystallographic orientations, there can be observed that the stiffness response of the indented material is effected not only by the type of material and its crystal orientation but also by the in-plane orientation of the Berkovich indenter (the axis of indenter rotation is perpendicular to material surface). From the non-axisymmetric nature of the Berkovich indenter it follows that the resistance of the materials against indenter penetration is different under the edge and under the face of the pyramid. It can be demonstrated in Fig. 4, where the normalized indentation moduli in $\langle 111\rangle$ direction are plotted against the indenter orientation for all studied materials. The normalization is based on the average modulus from all orientations. The effect is the most intense for highly anisotropic $\beta$-brass, for which indentation modulus range is about $5 \%$ of the averaged value. Moreover, the indenter orientation influenced also the contact area at the same penetration depth (Fig. 5).

For next plots, the effect of the indenter orientation was neglected and only averaged values for each crystallographic direction are presented. Indentation moduli for all 24 computed crystallographic orientations are presented in the form of modified inverse pole figures, where colours represent the level of indentation moduli $M$ : Fig. 6a illustrates tungsten, Fig. 6b - AISI 304 steel and Fig. $6 c-\beta$-brass. All three figures show the same trend - from the softest direction $\langle 001\rangle$ through relatively hard $\langle 110\rangle$ to the hardest $\langle 111\rangle$. The degree of anisotropy influences the difference between the hardest

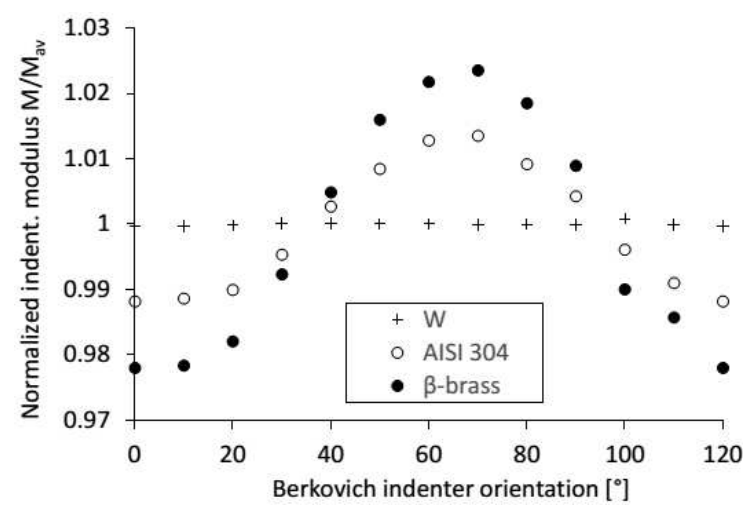

Fig. 4. The effect of the indenter angle on normalized indentation moduli for $\langle 111\rangle$ crystal orientation and materials with various degree of anisotropy.

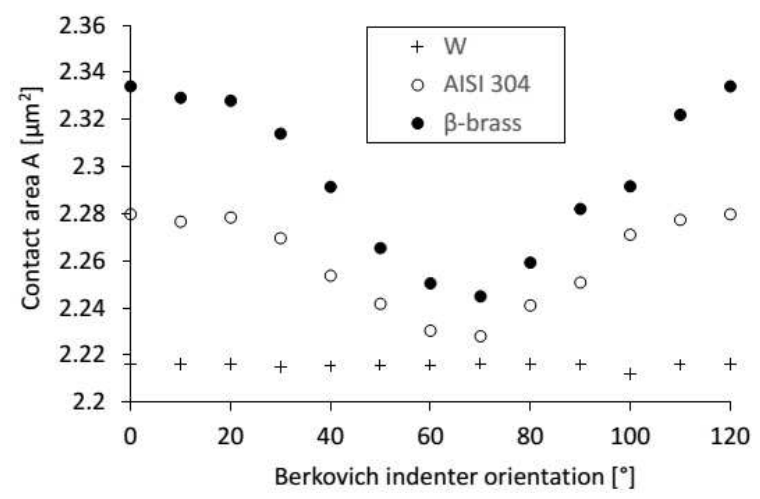

Fig. 5. The effect of the indenter angle on contact area for $\langle 111\rangle$ crystal orientation and materials with various degree of anisotropy.

and the softest directions, as can be seen in Fig. 7. But the difference is much lower than that defined by the $E_{111} / E_{100}$ ratio (in Fig. 7 are the Young moduli normalized by the polycrystalline modulus obtained from elastic constants according to Kröner-Eshelby model). This is caused by the complex, triaxial nature of the stress field under the indenter, which reduces the uniaxial anisotropy ratio by the factor of 6 for the $\beta$-brass and by 2.5 for SS.

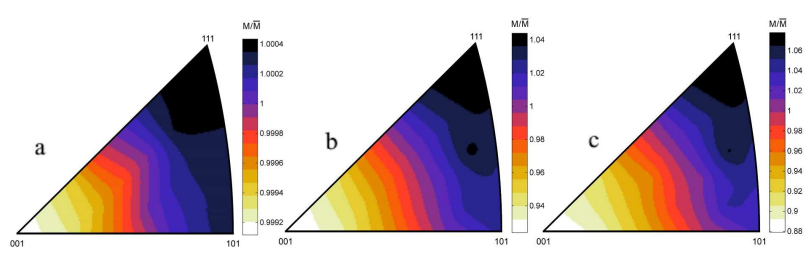

Fig. 6. Normalized indentation modulus for various crystallographic orientations of 1) tungsten, 2) SS, 3) $\beta$-brass. 


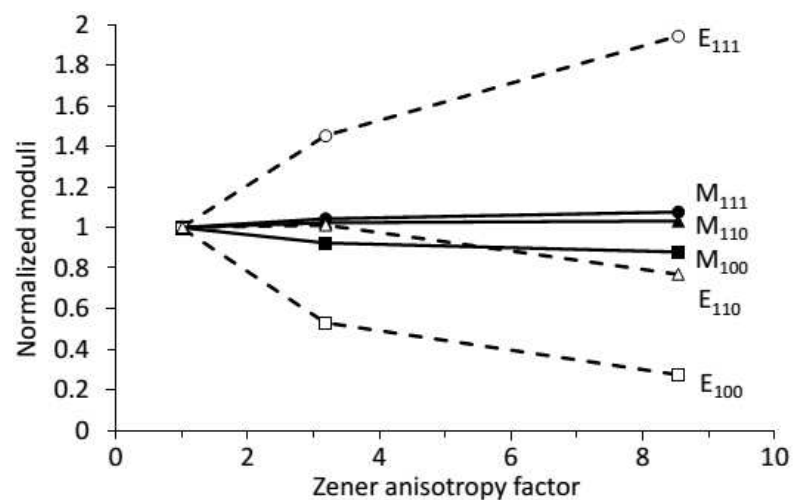

Fig. 7. Comparison of the normalized values of indentation and the Young moduli in various crystallographic orientations.

\section{Conclusions}

The effect of the degree of anisotropy on indentation moduli and contact area under the Berkovich indenter were investigated by a set of finite element simulations for three concrete cubic materials: nearly isotropic tungsten, anisotropic SS and highly anisotropic $\beta$-brass. The achieved results led to the following conclusion:

- Indentation moduli are sensitive to in-plane orientation of the non-axisymmetric Berkovich indenter, but even the highest observed variation of $5 \%$ for $\beta$-brass is within the scatter of the experimental data in practical applications.
- The indentation moduli are correlated with the single grain orientation.

- Computed anisotropy ratios between the hardest and the softest directions are about 1.1-1.2 for common anisotropic materials and are much lower than that expected from uniaxial measurements. The more anisotropic material is, the higher discrepancy can be expected.

\section{Acknowledgments}

This work was supported by the Czech Science Foundation, grant No. GACR P108/12/1872 and by the Grant Agency of the Czech Technical University in Prague, grant No. SGS13/223/OHK4/3T/14.

\section{References}

[1] P. Haušild, A. Materna, J. Nohava, Key Eng. Mater. 586, 31 (2014).

[2] A. Materna, P. Haušild, Key Eng. Mater. 606, 65 (2014).

[3] W.C. Oliver, G.M. Pharr, J. Mater. Res. 7, 1564 (1992).

[4] W.C. Oliver, G.M. Pharr, J. Mater. Res. 19, 3 (2004).

[5] J.J. Vlassak, W.D. Nix, Philos. Mag. A 67, 1045 (1993).

[6] J.J. Vlassak, W.D. Nix, J. Mech. Phys. Solids 42, 1223 (1994).

[7] H.M. Ledbetter, Physica $B+C$ 128, 1 (1985). 